\title{
Substantive incidental cardiac findings revealed by non-cardiac CT examination of the thorax
}

\author{
M Durand, N Paul, A Crean \\ Joint Division of Medical Imaging, Peter Munk Cardiac Centre, Toronto General Hospital, Toronto, Canada \\ M Durand, MB ChB, FCRad (Diag) \\ N Paul, MD, MRCP, FRCR, FRCRC
}

Joint Division of Medical Imaging and Division of Cardiology, Peter Munk Cardiac Centre, Toronto General Hospital, Toronto, Canada A Crean, MD, MRCP, FRCR

Corresponding author: M Durand (mirandadurand@gmail.com)

\begin{abstract}
Limitations inherent in CT imaging of the thorax left the heart as an ill-defined and largely ignored area. The advent of multi-detector CT scanning and shortened imaging times has allowed clear identification of cardiac structures and pathology. Motion-free cardiac images require synchronisation of image acquisition with the cardiac cycle (cardiac 'gating' or 'triggering'). However, significant information can be obtained from careful scrutiny of ungated chest CT images, which may then direct further imaging or investigation. We review 5 cases demonstrating interesting cardiac findings identified on non-cardiac scans.
\end{abstract}

S Afr J Rad 2013;17(1):34-37. DOI:10.7196/SAJR.791

For years, the technological limitations inherent in CT imaging of the thorax meant that the heart was an ill-defined and largely ignored 'space-filler' between the lungs. With the advent of multi-detector CT scanning (MDCT) in the late 1990s, and shortened imaging times, it has now become possible to clearly identify cardiac structures and pathology. Motion-free cardiac images require synchronisation of image acquisition with the cardiac cycle (cardiac 'gating' or 'triggering'). However, significant information can often be gleaned from careful scrutiny of ungated chest CT images, which may then direct further imaging or investigation.

In the following examples, we review 5 cases demonstrating interesting cardiac findings identified on non-cardiac scans.

\section{Case 1}

\section{History}

A 26-year-old woman presented to her referring physician with a history of abdominal pain, generalised swelling and bloating. Her facial swelling was reported to be worse in the mornings, with subsequent improvement during the course of the day. She volunteered a history of congenital rubella. On examination, no ascites, hepatomegaly or peripheral oedema was identified. Her blood results showed normal liver function with bilirubin $8 \mu \mathrm{mol} / \mathrm{l}$, ALT 29 units/litre, AST 32 units/litre and ALP 92 units/litre. Her albumin, however, was slightly decreased at $26 \mathrm{~g} / \mathrm{l}$. An abdominal CT scan was ordered to assess her symptoms.

\section{Imaging}

Abdominal CT scan demonstrated a mildly enlarged liver with dilated hepatic veins, dilated inferior vena cava (Fig. 1a), and diffuse heterogeneous enhancement following administration of intravenous

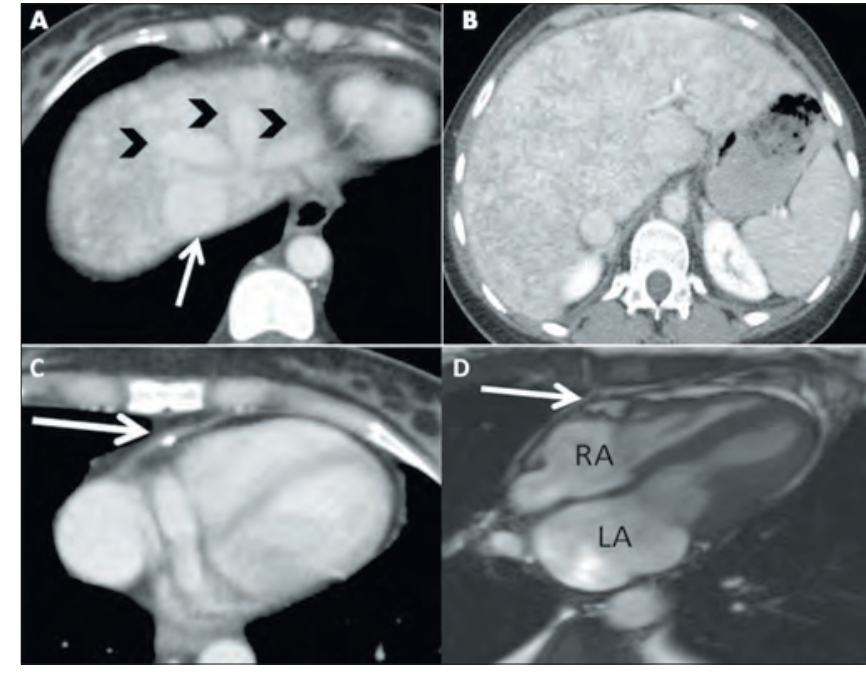

Fig. 1. Axial post contrast-enhanced CT scans of the abdomen in a 26-yearold woman with a history of abdominal pain, generalised swelling and bloating. (a) CT scan through the upper abdomen demonstrates dilated inferior vena cava (arrow) and hepatic veins (arrow heads). (b) CT scan through the upper liver demonstrates a mildly enlarged liver with diffuse heterogeneous enhancement in keeping with hepatic venous congestion. (c) Post contrast images through the lower chest on abdominal CT scan demonstrate some pericardial thickening with foci of pericardial calcification adjacent to the basal right ventricle and atrio-ventricular groove (arrow). (d) The four-chamber cine steady-state free precession (SSFP) cardiac MRI image demonstrates biatrial enlargement with small biventricular volumes. Pericardial thickening is also noted adjacent to the atrio-ventricular groove (arrow). Bilateral pleural effusions are also seen ( $L A=$ left atrium; $R A=$ right atrium). 
iodinated contrast medium (CM) (Fig. 1b) - all in keeping with hepatic venous congestion.

Limited images of the chest demonstrated pericardial thickening and calcification adjacent to the right ventricle (Fig. 1c). This raised the possibility of constrictive pericarditis, and a cardiac MRI was requested. This demonstrated small ventricles with relative bi-atrial enlargement (Fig. 1d). The pericardial thickening seen on the CT scan was confirmed on the MRI scan (Fig. 1d). The cine images demonstrated 'septal bounce' in keeping with constrictive physiology. The patient had invasive measurement of cardiac pressures which confirmed a constrictive physiology.

\section{Diagnosis}

Pericardial thickening and calcification with a restrictive physiology was the diagnosis. The pericardial thickening and calcifications were most likely due to previous pericarditis. It is possible that the patient might have developed this as a consequence of her rubella infection, ${ }^{[1]}$ although it would be unusual for her to present with symptoms so long after a congenital infection. It is more probable that her presentation resulted from a recent episode of subclinical pericarditis that resulted in pericardial calcification.

\section{Teaching point}

The normal pericardium measures less than $2-3 \mathrm{~mm}$. Pericardial thickening $>4 \mathrm{~mm}$ together with clinical features of predominantly rightsided cardiac failure are suggestive of constrictive physiology. While CT can be particularly useful in assessing pericardium thickness and calcifications, MRI is used to distinguish between constrictive pericardial disease and restrictive myocarditis. ${ }^{[2]}$ Septal bounce can be demonstrated on free breathing cine MRI images in patients with constrictive physiology, which distinguish it from restrictive cardiomyopathy. Note, however, that although pericardial constriction and restrictive cardiomyopathy are very different pathologies, they give rise to a final common physiological appearance of impaired diastolic ventricular filling - so-called 'restrictive filling pattern' or 'restrictive physiology'. Hence, even pericardial constriction presents with a restrictive physiology: a source of much diagnostic confusion and a reason why CT and MRI are so valuable.

\section{Case 2}

\section{History}

A 63-year-old man was referred to our department for follow-up after right upper and middle lobe lobectomy for lung cancer. Clinically, he was stable with no new symptoms. He volunteered a history of 3 myocardial infarctions after triple coronary bypass surgery in 1993.

\section{Imaging}

The transaxial images from thoracic MDCT demonstrated an aneurysm in the proximal (Fig. 2a) and distal aspects (Fig. 2b) of the saphenous venous graft to the right coronary artery (RCA), with mural thrombi in both aneurysms.

\section{Diagnosis}

Saphenous vein graft aneurysms were diagnosed. Although the distal aneurysm was closely related to the suture line, the proximal aneurysm was distal to the suture line. Both were felt to represent true aneurysms. Owing to the patient's underlying medical conditions, it was decided

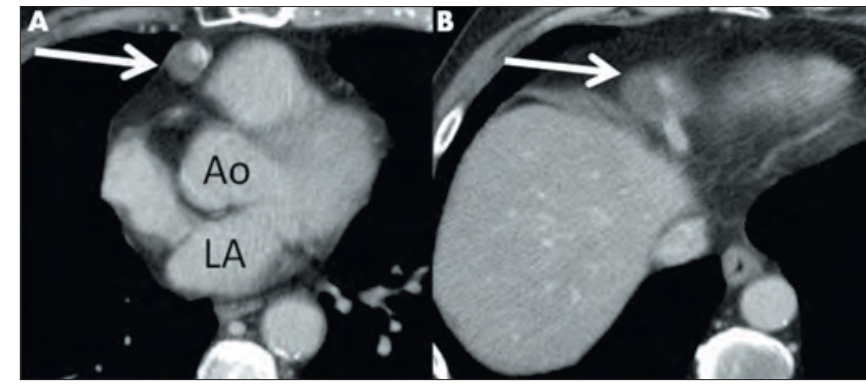

Fig. 2. Post contrast-enhanced chest CT scan in a 63-year-old man followed up post lobectomy for lung carcinoma. (a) Transaxial CT image at the level of the aortic valve demonstrates an eccentric mural thombus within an aneurysm of the proximal saphenous vein to right coronary artery graft (arrow) (Ao = aortic root; $L A=$ left atrium). (b) Transaxial CT image to the left of the distal saphenous vein right coronary artery graft demonstrates concentric mural thrombus within a second aneurysm at the implantation site of the graft (arrow).

to treat these aneurysms with percutaneous deployment of Amplatzer vascular plugs despite the associated risk of myocardial infarction.

\section{Teaching point}

Saphenous vein graft aneurysms are a rare complication of coronary bypass surgery and can be divided into true and pseudo-aneurysms. Pseudo-aneurysms are associated with technical complications at the suture line or infections. The distinction is academic as surgical resection and revascularisation are the preferred treatment for both. ${ }^{[3]}$

\section{Case 3}

\section{History}

A 67-year-old man was admitted to an outlying hospital with a late presentation of an anterior ST segment elevation myocardial infarction. At the time of admission, an attempted percutaneous coronary intervention was unsuccessful. Three days later, he was transferred to the ICU at our hospital with severe hypoxaemia and a clinical presentation suggestive of septic shock. A chest CT was requested to rule out pulmonary embolism or pneumonia.

\section{Imaging}

Thoracic MDCT demonstrated an area of hypoperfusion in the interventricular septum (Fig. 3a), suggesting an area of infarction, and a

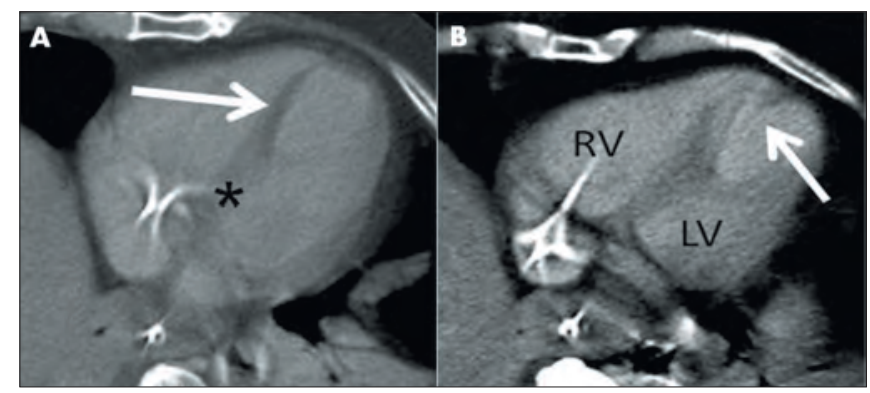

Fig. 3. Post contrast chest CT scan in a 67-year-old man performed to rule out pulmonary embolism or pneumonia. (a) Transaxial CT image at the level of the interventricular septum demonstrates hypoperfusion of the mid-distal septum (arrow) in comparison with the basal septum $\left(^{*}\right)$. (b)Transaxial CT image at the level of the inferior septum demonstrates a large defect in the muscular portion of the septum (arrow). ( $L V=$ left ventricle; $R V=$ right ventricle.) 


\section{CASE SERIES}

muscular ventricular septal defect (VSD) apical and inferior to this (Fig. 3b). Bilateral basal atelectasis was noted with no evidence for pulmonary embolism.

\section{Diagnosis}

Postmyocardial infarction ventricular septal defect was diagnosed. The patient had a myocardial infarction involving the muscular portion of the inter-ventricular septum complicated by a VSD. Myocardial perfusion isotope studies and stress perfusion cardiac MRI are often used to determine the extent of myocardial ischaemia and scarring in patients with ischaemic heart disease. It is less well appreciated that myocardial infarction can also be seen on even ungated MDCT as a region of myocardial hypoperfusion.

\section{Teaching point}

Ventricular septal rupture after myocardial infarction is a rare complication with a reported incidence of $1-2 \% \cdot{ }^{[4]}$ Rupture occurs 3 - 5 days after the myocardial infarction and the patient presents with sudden deterioration, new onset cardiac murmur, congestive heart failure, and often in cardiogenic shock. Treatment is initial stabilisation with inotropes and often an intraaortic balloon pump with subsequent surgical closure of the defect. There are, however, a few small case series reported in the literature where transcatheter closure of these defects has been successfully performed. ${ }^{[5]}$

\section{Case 4}

\section{History}

A 54-year-old man was referred for an abdominal CT for suspected cardiogenic liver cirrhosis. On examination, the patient had generalised oedema, and abdominal distention with mild ascites. The patient had had congenital valvular heart disease and had undergone several pulmonary valve surgeries since childhood. His pulmonary valve was replaced with a porcine root in 2002.

\section{Imaging}

The abdominal images revealed chronic passive liver congestion with features of hepatic cirrhosis. Venous phase axial images through

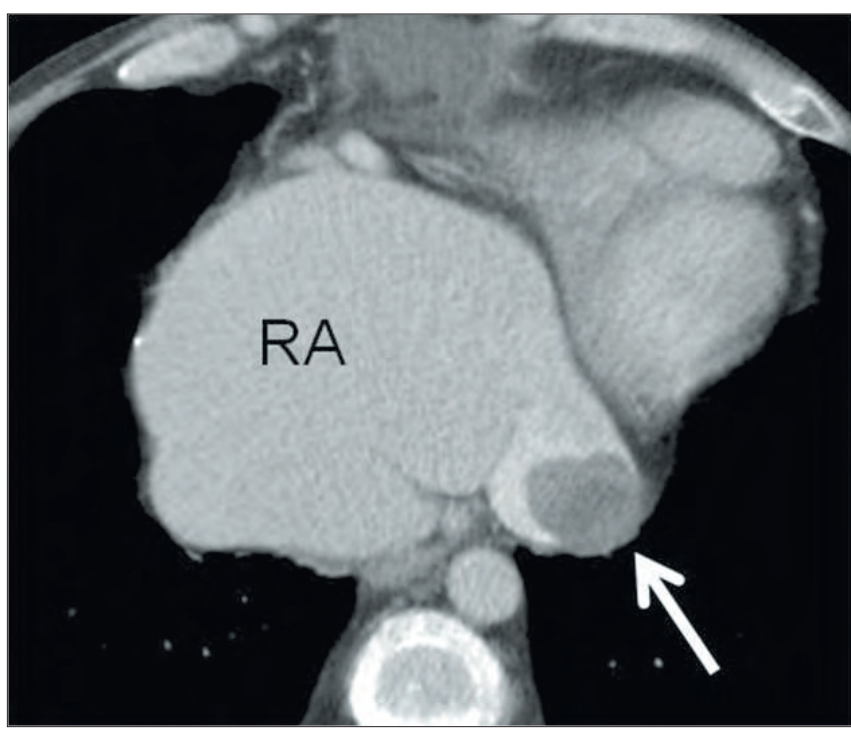

Fig. 4. Venous phase transaxial image from a CT scan in a 54-year-old man demonstrates a filling defect in the coronary sinus in keeping with a thrombus. the lower chest demonstrated a filling defect in the coronary sinus (Fig. 4).

\section{Diagnosis}

Coronary sinus thrombosis was diagnosed. The patient had a coronary sinus thrombosis that was subsequently resected during surgical replacement of the mitral valve and down-sizing of the right atrium.

\section{Teaching point}

A few cases of primary coronary sinus thrombosis have been reported ${ }^{[6]}$ but coronary sinus thrombosis is mostly associated with previous invasive cardiac procedures such as central venous line placement, insertion of pacing wires, or coronary sinus catheterisation for ventricular lead placement during cardiac resynchronisation therapy. [7] This patient had had several previous invasive cardiac procedures as well as a severely dilated right atrium, both predisposing to thrombosis formation.

\section{Case 5}

\section{History}

A 65-year-old man, under disease surveillance for metastatic gastric carcinoma, was sent for routine chest, abdomen and pelvis CT. The patient had completed one cycle of chemotherapy 18 months previously and was clinically well with no new symptoms.

\section{Imaging}

The venous phase chest CT demonstrated a mass lesion in the inferior aspect of the right ventricle (Fig. 5a). This was better appreciated on the sagittal reconstructed images of the chest (Fig. 5b). Cardiac MRI was performed to assess contrast enhancement of the lesion to determine

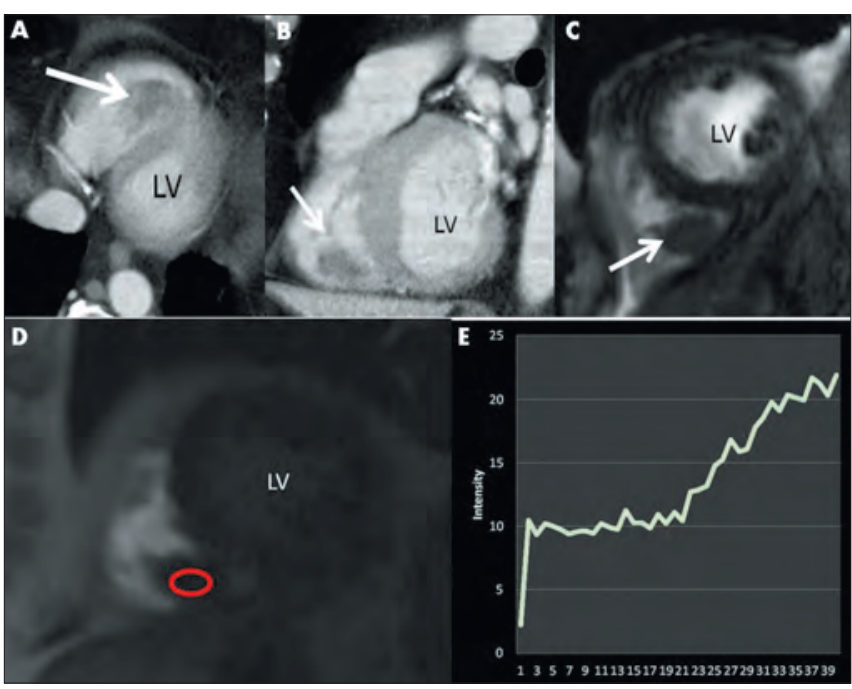

Fig. 5. Post contrast venous phase chest CT scan in a 65-year-old man with gastric carcinoma. (a) Transaxial images demonstrate a filling defect (arrow) in the basal right ventricle ( $L V=$ left ventricle). (b) Post contrast-enhanced sagittal reconstructed image of the chest demonstrates a filling defect in the basal right ventricle ( $L V=$ left ventricle). (c) Cardiac MRI steady-state free precession image obtained in a short axis orientation demonstrates a mass lesion in the basal right ventricle (arrow) ( $L V=$ left ventricle). (d) The right ventricular mass demonstrates gadolinium enhancment during perfusion imaging as demonstrated on (e) the enhancement curve display on the right. 


\section{CASE SERIES}

whether this was a thrombus or intracardiac mass. The mass (Fig. 5c) demonstrated gadolinium enhancement on the perfusion images (Figs $5 \mathrm{~d}$ and $\mathrm{e})$.

\section{Diagnosis}

Enhancing right atrial mass was diagnosed; the mass most probably represents a metastasis from the known gastric carcinoma.

\section{Teaching point}

Cardiac masses are uncommon but, when they do occur, a metastatic origin is by far the most common cause. MRI first-pass perfusion imaging with gadolinium is often used to distinguish between thrombus and masses. Conventionally, thrombus does not enhance with gadolinium; however, large chronic thrombi may occasionally have peripheral enhancement, making differentiation between mass and thrombus challenging.$^{[8]}$ Typically, tumours demonstrate at the least partial uptake of gadolinium unless very avascular. The most common tumors metastasising to the heart are breast, kidney, oesophagus, lymphoma, leukaemia and melanoma. ${ }^{[8]}$

1. Fink C, Schaad UB, Stocker FP. Perikarditis als Komplikation von Roteln. [Pericarditis as a complication of rubella]. Schweizerische Medizinische Wochenschrift. 1987;117(1):28-32

2. Wang ZJ, Reddy GP, Gotway MB, Yeh BM, Hetts SW, Higgins CB. CT and MR imaging of pericardial disease. Radiographics 2003;23 Spec No:S167-180.

3. Kalimi R, Palazzo RS, Graver LM. Giant aneurysm of saphenous vein graft to coronary artery compressing the right atrium. Ann Thorac Surg 1999;68(4):1433-7143. [http://dx.doi.org/10.1016/S00034975(99)00848-6]

4. Crenshaw BS, Granger CB, Birnbaum Y, et al. Risk factors, angiographic patterns, and outcomes in patients with ventricular septal defect complicating acute myocardial infarction. GUSTO-I (Global Utilization of Streptokinase and TPA for Occluded Coronary Arteries) Trial Investigators. Circulation 2000;101(1):27-32. [http://dx.doi.org/10.1161/01.CIR.101.1.27]

5. Moreno N, Silva JC, Andrade A. Complicated transcatheter closure of postinfarction ventricular acute septal defect. J Invasive Cardiol 2011;23(10):E244-246.

6. Ramsaran EK, Sadigh M, Miller D. Sudden cardiac death due to primary coronary sinus thrombosis. South Med J 1996;89(5):531-533. [http://dx.doi.org/10.1097/00007611-199605000-00019]

7. Parmar RC, Kulkarni S, Nayar S, Shivaraman A. Coronary sinus thrombosis. J Postgrad Med 2002;48(4):312-313.

8. O'Donnell DH, Abbara S, Chaithiraphan V, et al. Cardiac tumors: optimal cardiac MR sequences and spectrum of imaging appearances. AJR 2009;193(2):377-387. [http://dx.doi.org/10.2214/AJR.08.1895] 